\title{
МОРФОМЕТРИЧЕСКИЙ АНАЛИЗ СЛОЖНЫХ КОМБИНАЦИОННЫХ ФОРМ КРИСТАЛЛОВ ТРИГОНАЛЬНОЙ СИНГОНИИ
}

Страшненко Г.И.

https://doi.org/10.31241/MIEN.2018.14.13

Екатеринбург, strashnenko_gi@mail.ru

В пяти классах симметрии тригональной сингонии возможны 10 простых кристаллографических форм (ПКФ), половина из которых (дитригональная и гексагональная бипирамиды, ромбоэдр, скаленоэдр, трапецоэдр) представлена закрытыми переменными формами (табл. 1).

Таблица 1. Распределение простых кристаллографических форм (ПКФ) по классам симметрии тригональной сингонии по М.П. Шаскольской [8].

\begin{tabular}{|c|c|c|c|c|c|}
\hline \multirow{2}{*}{ 卢 } & \multicolumn{5}{|c|}{ Классы симметрии простых форм } \\
\hline & 3 & $\overline{3}$ & $3 \mathrm{~m}$ & 32 & $\overline{3} m$ \\
\hline$h k . l$ & 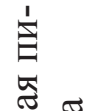 & f & $\begin{array}{c}\text { Дитригональная } \\
\text { пирамида }\end{array}$ & $\begin{array}{c}\text { Тригональный } \\
\text { трапецоэдр }\end{array}$ & $\begin{array}{l}\text { Тригональный } \\
\text { скаленоэдр }\end{array}$ \\
\hline h0.1 & 蛋莺 & 离 & $\begin{array}{c}\text { Тригональная } \\
\text { пирамида }\end{array}$ & \multicolumn{2}{|c|}{ Ромбоэдр } \\
\hline hh.1 & ڤ్ & & $\begin{array}{c}\text { Гексагональная } \\
\text { пирамида }\end{array}$ & $\begin{array}{l}\text { Тригональная } \\
\text { бипирамида }\end{array}$ & $\begin{array}{c}\text { Гексагональная } \\
\text { бипирамида }\end{array}$ \\
\hline hk.0 & \multirow{3}{*}{ 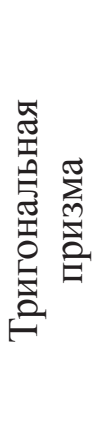 } & \multirow{3}{*}{ 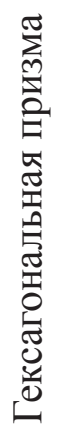 } & \multicolumn{2}{|c|}{ Дитригональная призма } & $\begin{array}{c}\text { Дигексагональная } \\
\text { призма }\end{array}$ \\
\hline 10.0 & & & $\begin{array}{c}\text { Тригональная } \\
\text { призма }\end{array}$ & \multicolumn{2}{|c|}{ Гексагональная призма } \\
\hline 11.0 & & & $\begin{array}{c}\text { Гексагональная } \\
\text { призма }\end{array}$ & $\begin{array}{c}\text { Тригональная } \\
\text { призма }\end{array}$ & $\begin{array}{c}\text { Гексагональная } \\
\text { призма }\end{array}$ \\
\hline 00.1 & 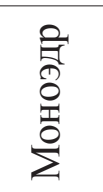 & 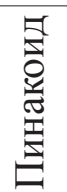 & Моноэдр & & гакоид \\
\hline
\end{tabular}

Наиболее сложные формы кристаллов с участием ромбоэдров, дитригональных скаленоэдров и тригональных трапецоэдров широко распространены в двух классах симметрии этой сингонии 32 и $\overline{3} m$. Рассмотрим некоторые комбинации этих ПКФ, аналитически выведем в общем виде уравнения их морфометрических функций, а в качестве примера для конкретных минералов построим морфометрические и морфогенетические диаграммы. 


\section{Комбинация гексагональной призмы $\{10.1\}$ и двух коррелятивных ромбоэдров $\{\mathrm{h} 0 . l\}$ и $\{0 \mathrm{~h} . l\}$}

Комбинация гексагональной призмы и двух коррелятивных ромбоэдров возможна в двух классах симметрии 32 и $\overline{3} m$. Эта комбинация характерна для кристаллов кварца и кальцита, одних из наиболее распространенных в мире кристаллов, сыгравших огромную роль в развитии науки и техники XX века. Габитус кристаллов, образованных комбинацией этих ПКФ, зависит от двух показателей: удлинения гексагональной призмы $x$ и отношения площадей граней коррелятивных ромбоэдров ( $\eta)$. Эти показатели примем за аргументы морфометрической функции рассматриваемой комбинации. В качестве параметра приведения [4] выберем длину стороны гексагонального сечения призмы и обозначим её символом $c$. Тогда показатели габитуса кристаллов определятся следующими выражениями:

$$
\begin{gathered}
x=l_{m} / 2 c \\
\eta=\frac{S_{-r}}{S_{+r}}=\varphi(y),
\end{gathered}
$$

где $l_{m}$ - длина призматической части кристалла;

$S_{+r} ; S_{-r}$ - площадь грани соответственно положительного и отрицательного ромбоэдра;

$y$ - относительная доля ребра между двумя смежными гранями ромбоэдра одного знака в общей его длине, возможной в случае моноромбоэдрического габитуса головок кристалла. На рисунке 1 этот аргумент равен отношению отрезков $(d k) /(d g)$.

На рисунке 1 изображены проекции головки кристалла на XY-плоскость (ряд $a$ ), вертикальный разрез кристалла по плоскости (11.0) (ряд б), форма и ориентировка относительно центра кристалла граней призмы (ряд в). При $y=0 \eta=1.0$ габитус кристаллов диромбоэдрический или псевдогексагональный (столбец I на рис. 1), а при $y=1 \eta=0$ моноромбоэдрический (столбец III на рис.1), Между этими двумя крайними габитусами существует непрерывный ряд промежуточных форм кристалла (один из них изображен на рисунке 1 в столбце II). Выведем формулу морфометрической функции ( $F$-функции), описывающей данный ряд. Запишем формулу $F$-функции в общем виде $/ 3 /$ :

$$
F=V^{-\frac{2}{3}} M,
$$

где $V$ и $M$ - соответственно объем и площадь полной поверхности кристалла.

Сначала найдем уравнения $F$-функции для крайних членов ряда. Для этого объем и полную поверхность кристалла определим через параметр приведения $c$ (длину стороны гексагонального сечения призмы), угловые 
константы кристалла и принятые переменные $(x, y)$. Обозначим угловые константы кристалла:

- $\rho$ - полярная координата граней ромбоэдров (для коррелятивных форм эти координаты равны);

- $\alpha$ - полярная координата ребра между двумя смежными гранями одного ромбоэдра.

Кристалл псевдогексагонального габитуса геометрически представляет комбинацию гексагональной призмы и гексагональной бипирамиды. Объем такого кристалла равен сумме объема призмы $\left(V_{p r}\right)$ и удвоенного объема пирамиды $\left(V_{p i}\right)$ :

$$
V=V_{p r}+2 V_{p i}=S_{6} \cdot 2 c x+2 \frac{1}{3} S_{6}(d e)=2 S_{6}\left(c x+\frac{(d e)}{3}\right)=2 \frac{3 \sqrt{3}}{2} c^{2}\left(c x+\frac{\frac{\sqrt{3} c}{2} \operatorname{tg} \rho}{3}\right)=c^{3} 3 \sqrt{3}\left(x+\frac{\operatorname{tg} \rho}{2 \sqrt{3}}\right)
$$

где $S_{6}$ - площадь гексагонального сечения призмы.

Запишем полученное выражение для объема кристалла в случае I.

$$
V^{I}==c^{3} 3 \sqrt{3}\left(x+\frac{\operatorname{tg} \rho}{2 \sqrt{3}}\right)
$$

Полная поверхность кристалла равна сумме площади граней призмы $\left(S_{p r}\right)$ и удвоенной площади боковой поверхности пирамиды $\left(S_{p i}\right)$

$$
M=S_{p r}+2 S_{p i}=12 c^{2} x+2 \frac{S_{6}}{\operatorname{Cos} \rho}=\left(12 c^{2} x+2 \frac{\frac{3 \sqrt{3}}{2} c^{2}}{\operatorname{Cos} \rho}\right)=c^{2} 3\left(4 x+\frac{\sqrt{3}}{\operatorname{Cos} \rho}\right)
$$

Перепишем полученное выражение без вывода.

$$
M^{I}=c^{2} 3\left(4 x+\frac{\sqrt{3}}{\operatorname{Cos} \rho}\right)
$$

Подставив (4) и (5) в (3) получим формулу $F$-функции рассматриваемой комбинации ПКФ для частного случая равного развития положительного и отрицательного коррелятивных ромбоэдров.

$$
F=\left[3 \sqrt{3}\left(x+\frac{\operatorname{tg} \rho}{2 \sqrt{3}}\right)\right]^{-\frac{2}{3}} 3\left(4 x+\frac{\sqrt{3}}{\operatorname{Cos} \rho}\right)
$$



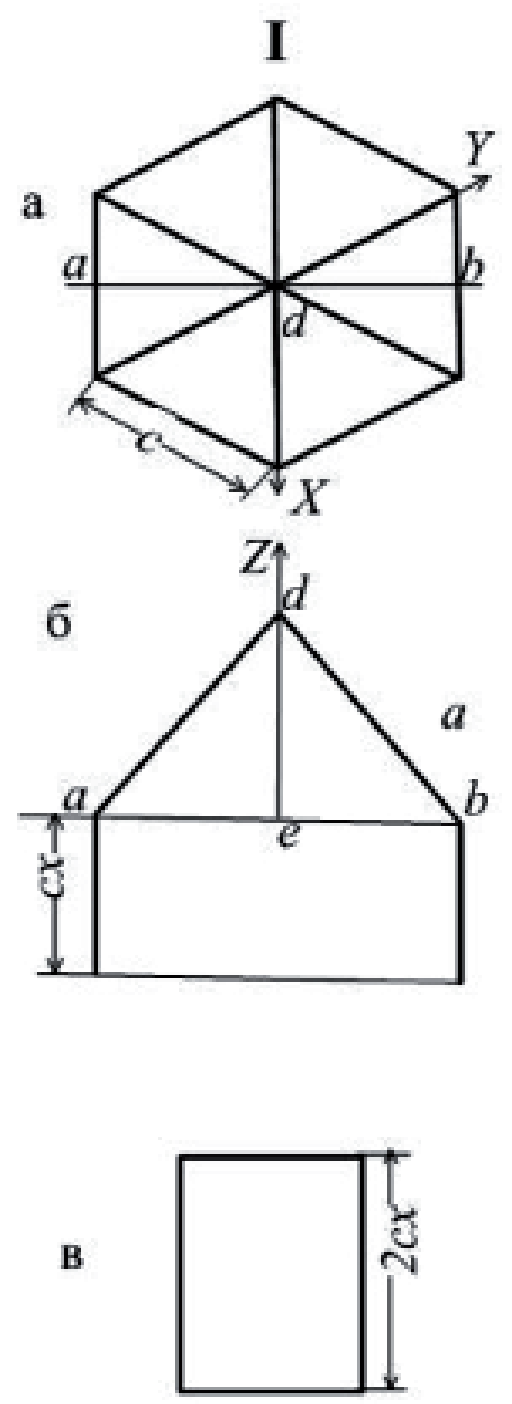
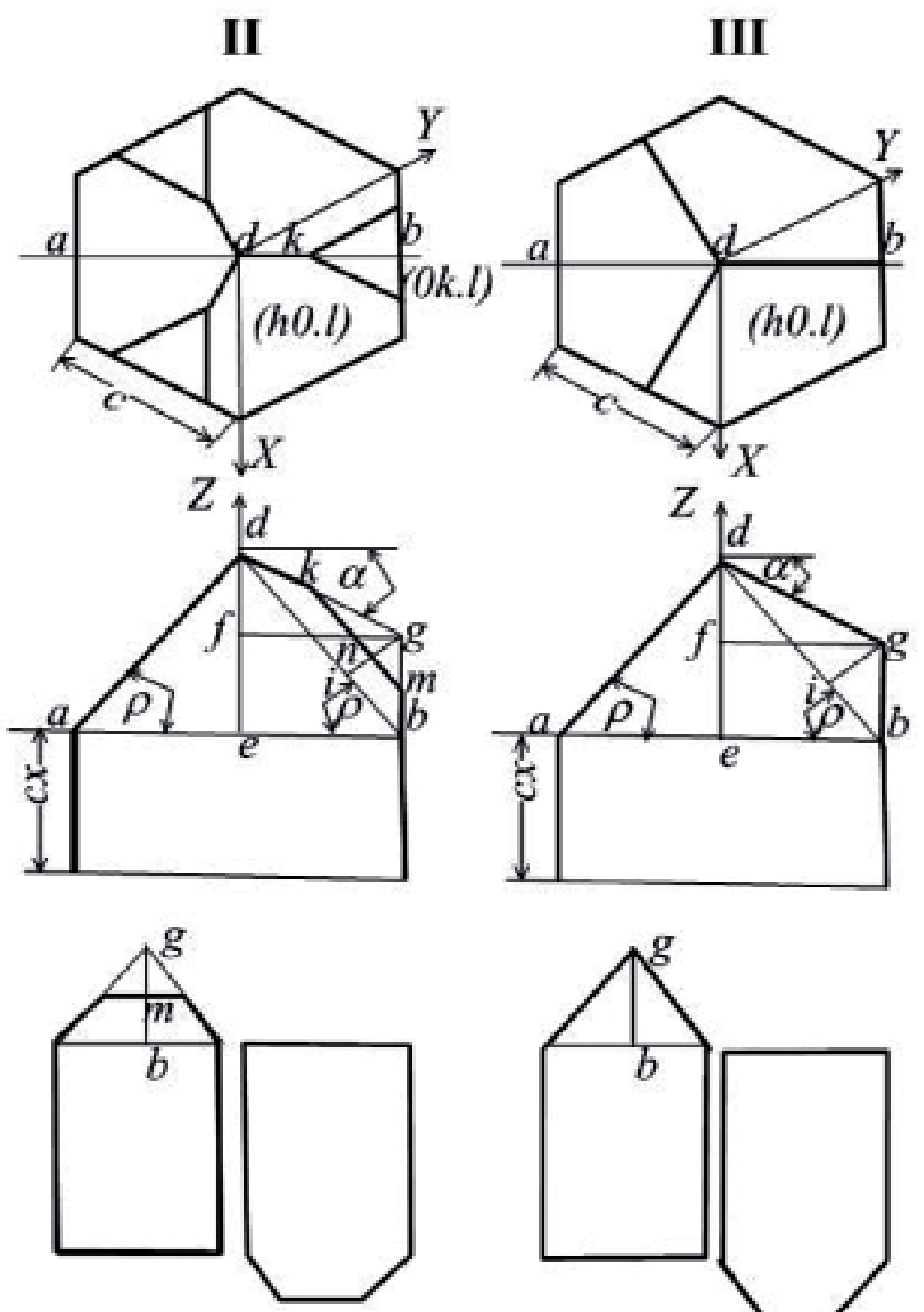
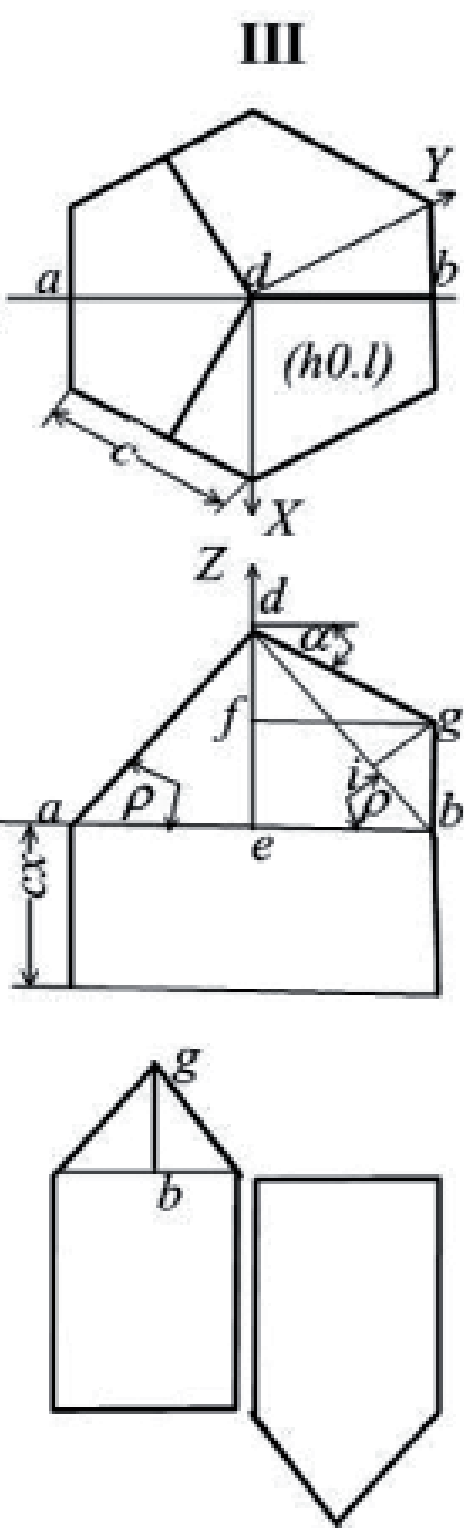

Рис. 1. Возможные габитусы кристаллов, образованных комбинаиией гексагональной призмы и двух коррелятивных ромбоэдров.

В равной степени формула (6) описывает комбинацию гексагональной призмы и гексагональной бипирамиды [5], встречающуюся в классе $\overline{3} m$ тригональной сингонии (табл. 1) и в классах $6 / \mathrm{m}, 622,6 / \mathrm{mmm}, \overline{6} \mathrm{~m} 2$ гексагональной сингонии [9].

Далее выведем формулу $F$ - функции для частного случая моноромбоэдрического габитуса (III). В сравнении со случаем I объем кристалла возрастает на 6 объемов трехгранной пирамиды, нарастающей на гранях отрицательного ромбоэдра. Площадь основания этой пирамиды $\left(S_{3}\right)$ равна площади грани ромбоэдра в случае I, а высота равна отрезку $(g i)$. Площадь полной поверхности кристалла при этом возрастает на 6 площадей треугольника, венчающего одну из горизонтальных сторон грани призмы (рис. 1, IIІв). Поскольку мы имеем комбинацию с коррелятивными ромбоэдрами, площадь боковой поверхности головки кристалла при всех изменениях соотношени- 
ях их долей в огранении кристалла остается постоянной. Объем и полную поверхность кристалла для первого случая обозначим символами $V^{I} u M^{I}$. Тогда

$$
\begin{aligned}
& V^{I I I}=V^{I}+6 \frac{1}{3} S_{3}(g i)=V^{1}+2 \frac{\sqrt{3}}{4 \operatorname{Cos} \rho} c^{2} \cdot(d g) \operatorname{Sin}(\rho-\alpha)=V^{I}+c^{2} \frac{\sqrt{3}}{2 \operatorname{Cos} \rho} \frac{(f g) \operatorname{Sin}(\rho-\alpha)}{\operatorname{Sin}\left(90^{\circ}-\alpha\right)}=- \\
& \rightarrow V^{I}+c^{2} \frac{\sqrt{3}}{2 \operatorname{Cos} \rho} \frac{\sqrt{3} c \operatorname{Sin}(\rho-\alpha)}{2 \operatorname{Cos} \alpha}=V^{I}+c^{3} \frac{3 \operatorname{Sin}(\rho-\alpha)}{4 \operatorname{Cos} \rho \operatorname{Cos} \alpha}
\end{aligned}
$$

Подставив (4) в полученное выражение, получим формулу объема для случая III.

$$
\begin{gathered}
V^{I I I}=c^{3}\left[3 \sqrt{3}\left(x+\frac{\operatorname{tg} \rho}{2 \sqrt{3}}\right)+\frac{3 \operatorname{Sin}(\rho-\alpha)}{4 \operatorname{Cos} \rho \operatorname{Cos} \alpha}\right] \\
M^{I I I}=M^{I}+6 \frac{1}{2} c \cdot(g b)=M^{I}+3 c \cdot \frac{(g i)}{\operatorname{Sin}\left(90^{\circ}-\rho\right)}=M^{I}+c^{2} \frac{3 \sqrt{3} \operatorname{Sin}(\rho-\alpha)}{2 \operatorname{Cos} \alpha \operatorname{Cos} \rho}
\end{gathered}
$$

Подставив (5) в полученное выражение, получим формулу площади полной поверхности кристалла для случая III.

$$
M^{I I I}=c^{2}\left[3\left(4 x+\frac{\sqrt{3}}{\operatorname{Cos} \rho}\right)+\frac{3 \sqrt{3} \operatorname{Sin}(\rho-\alpha)}{2 \operatorname{Cos} \alpha \operatorname{Cos} \rho}\right]
$$

Подставив (7) и (8) в (3), получим формулу $F$-функции рассматриваемой комбинации ПКФ для частного случая полного вырождения отрицательного ромбоэдра.

$$
F^{I I I}==\left[3 \sqrt{3}\left(x+\frac{\operatorname{tg} \rho}{2 \sqrt{3}}\right)+\frac{3 \operatorname{Sin}(\rho-\alpha)}{4 \operatorname{Cos} \rho \operatorname{Cos} \alpha}\right]^{-\frac{2}{3}}\left[3\left(4 x+\frac{\sqrt{3}}{\operatorname{Cos} \rho}\right)+\frac{3 \sqrt{3} \operatorname{Sin}(\rho-\alpha)}{2 \operatorname{Cos} \rho \operatorname{Cos} \alpha}\right]
$$

Перейдем к выводу общей формулы $F$-функции для рассматриваемого ряда габитусных форм (случай II). За основу примем $F$-функцию (9). Используем метод усечения пирамид, надстроенных на гранях отрицательного ромбоэдра (рис. 1, II б, в). В этом случае объем надстраиваемой пирамиды уменьшится на величину, пропорциональную $(1-y)^{3}$, а площадь граней призмы на величину, пропорциональную $(1-y)^{2}$. Определение переменной $y$ дано выше. Формула $F$-функции при этом примет вид (вывод формулы упускаем, он столь же геометрически прост, как и для случая III):

$$
F=\left[3 \sqrt{3}\left(x+\frac{\operatorname{tg} \rho}{2 \sqrt{3}}\right)+\frac{3 \operatorname{Sin}(\rho-\alpha)}{4 \operatorname{Cos} \rho \operatorname{Cos} \alpha}\left[1-(1-y)^{3}\right]\right]^{-\frac{2}{3}}\left[3\left(4 x+\frac{\sqrt{3}}{\operatorname{Cos} \rho}\right)+\frac{3 \sqrt{3} \operatorname{Sin}(\rho-\alpha)}{2 \operatorname{Cos} \rho \operatorname{Cos} \alpha}\left[1-(1-y)^{2}\right]\right]
$$


Формулу (10) можно выразить через $\eta$ произведя замену переменной $y$. воспользовавшись отношением: $\eta=1$ nри $y=0 ; \eta=0$ nри $y=1$.

$$
\eta=1-y
$$

Подставив (11) в (10), получим в общем виде уравнение морфометрической функции рассматриваемой комбинации ПКФ.

$$
F=\left[3 \sqrt{3}\left(x+\frac{\operatorname{tg} \rho}{2 \sqrt{3}}\right)+\frac{3 \operatorname{Sin}(\rho-\alpha)}{4 \operatorname{Cos} \rho \operatorname{Cos} \alpha}\left[1-\eta^{3}\right]\right]^{-\frac{2}{3}}\left[3\left(4 x+\frac{\sqrt{3}}{\operatorname{Cos} \rho}\right)+\frac{3 \sqrt{3} \operatorname{Sin}(\rho-\alpha)}{2 \operatorname{Cos} \rho \operatorname{Cos} \alpha}\left[1-\eta^{2}\right]\right]
$$

При $\eta=0$ формула (12) преобразуется к виду (9), а при $\eta=1$ к виду (6). Она описывает любые формы кристаллов, образованные рассмотренной комбинацией ПКФ.

Для примера воспользуемся данной формулой для построения морфометрической и морфогенетической диаграмм кристаллов кварца. Заметим, что нижеследующий пример, как и другие примеры данной статьи, преследуют цель продемонстрировать возможности использования предложенного метода морфометрического анализа для решения научно-практических задач минералогической кристаллографии. Все полученные при этом генетические выводы касательно конкретных месторождений носят предварительный и в большой степени демонстративный характер.

\section{Пример 1.}

Кварц $\mathrm{SiO}_{2}$. Тригонально-трапецоэдрический к.с., $a_{0}=4,91, c_{0}=5,40 \stackrel{0}{A}$. Угловые координаты грани $\{10.1\}: \rho=51.8^{\circ} ; \alpha=26.54^{\circ}$. На рисунке 2 изображены ортогональные проекции трех кристаллов кварца [2], соответствующие трем выше рассмотренным случаям. На чертежах показаны измеряемые линейкой геометрические элементы кристаллов и формулы, по которым рассчитываются переменные в (12). На практике форма кристаллов часто отклоняется от идеальной. В этом случае можно использовать среднее из величин трех однотипных элементов, но лучше для измерений подбирать кристаллы правильной формы.

Преобразуем формулу (12) применительно к кварцу, подставив входящие в неё все постоянные величины и произведя необходимые вычисления.

$$
F=\left\{5.2 x+1.91+0.48\left[1-\eta^{3}\right]\right\}^{-\left(\frac{2}{3}\right)}\left\{12 x+8.4+1.65\left[1-\eta^{2}\right]\right\}
$$

Морфометрическая диаграмма кварца, построенная по формуле (13), для разных фиксированных значений удлинения призматического пояса кристаллов, представлена на рисунке 3. Можно видеть, что при равных значениях удлинения морфометрическая функция кристаллов убывает в ряду от моноромбоэдрического до псевдогексагонального габитуса. С увеличением удлинения кристалла влияние доминирования положительного ромбоэдра на величину F-функции постепенно нивелируется. На диаграмме это видно по уменьшению наклона графиков к оси абсцисс с ростом параметра $x$ 


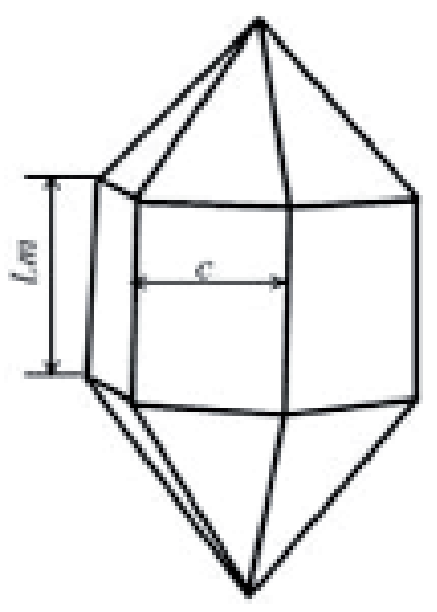

$x=L w / 2 c$ $y=0$

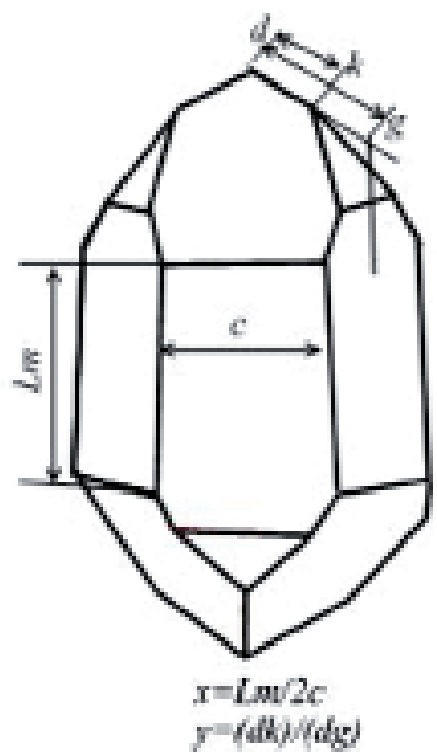

$y$ (da)dy

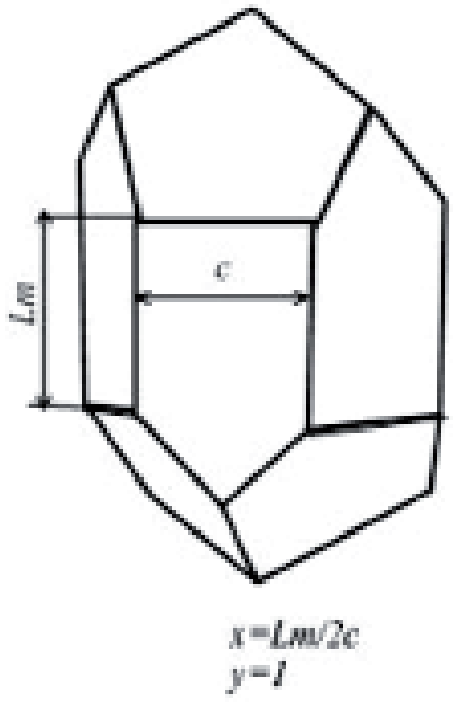

Рис. 2. Измеряемые элементы формы кристаллов и расчетные формулы переменных в уравнениях (10-13).

Использование морфометрической диаграммы покажем на примере решения конкретной задачи. Сформулируем минерагеническую задачу: сравнить проявления горного хрусталя Горбуновское, Ольховочное и Вандаманга с Астафьевским месторождением по признаку « форма кристаллов квар-

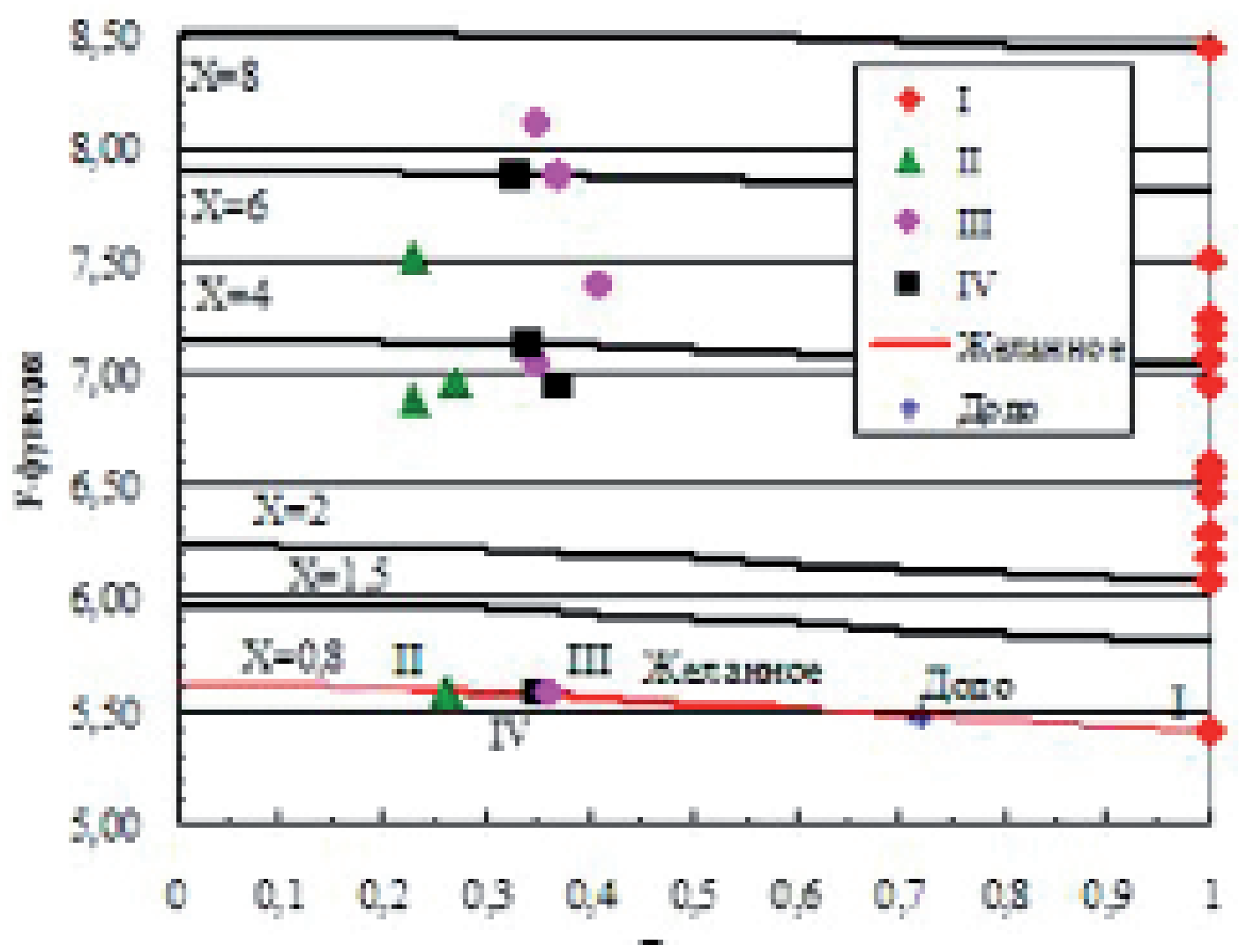

Рис. 3. Морфометрическая диаграмма кристаллов квариа.

I- Астафьевское месторождение (Ю. Урал), хрусталепроявления: II - Горбуновское (Ср. Урал), III - Ольховочное (С. Урал), IV-Вандаманга (Мадагаскар). 
ца». Для решения поставленной задачи произведем обмер кристаллов из сравниваемых объектов, как это показано на рисунке 2. Далее для каждого кристалла определим величины переменных и подставив их в уравнение (13), вычислим значение F-функции. Результаты расчетов, сведенные в таблицу 2, вынесем на диаграмму в виде маркеров определенной формы. Если судить по величине F-функции, как интегральной мере формы кристалла [4], то сравниваемые объекты не различаются по исследуемому признаку. Удлинение кристаллов $(x)$ в пределах одного месторождения или проявления и даже в одной друзе колеблется в широких пределах и оказывает существенное влияние на величину F-функции. Экспериментально доказано, что нормальная скорость роста граней призмы на порядок ниже, чем скорость роста граней ромбоэдров [1]. Природный процесс, протекающий при значительно меньших пересышениях, чем в эксперименте, приводит к полному торможению роста граней призмы. Объем кристаллов кварца сложен пирамидами нарастания граней одного или двух ромбоэдров. Следовательно, удлинение кристаллов кварца зависит исключительно от филогенических взаимоотношений индивидов в зоне геометрического отбора, а также от времени работы минералообразующей системы и не зависит от её термодинамических параметров.

Вместе с тем по величине участия отрицательного ромбоэдра в огранении кристалла $(\eta)$ оцениваемые проявления сходные между собой и отличаются от месторождения. Для каждого из проявлений колебание параметра $\eta$ практически не превышает ошибки измерений. Чтобы выделить влияние переменной $\eta$ на величину F-функции, минимизируем вклад в неё гексагональной призмы. Для этого приведем удлинение призматического пояса кристаллов к единой величине $x=0.8$. При таком удлинении F-функция комбинации гексагональной призмы и пинакоида принимает минимальное значение. На диаграмме (рис. 3) график F-функции с удлинением $x=0.8$ показан тонкой красной кривой. На кривой маркерами показаны рассматриваемые объекты. Дополнительно на неё вынесены трек (на графике $x=0.8$ показан утолщенным отрезком) и маркер полярноуральских месторождений Желанное и Додо, по которым значения параметра $\eta$ предоставлены автору исследователем этих месторождений Г.И. Крыловой. По её данным степень гексагональности $(\eta)$ кристаллов месторождения Желанное колеблется от 0.23 до 0.58 , для месторождения Додо она равна 0.72.

Морфометрический график с приведением удлинения кристаллов к единой величине показывает на существенное различие сравниваемых объектов по величине F-функции. Маркеры проявлений расположились на значительном удалении от Астафьевского месторождения в начальной половине трека месторождения Желанное, характеризующейся более низкими значениями переменной $\eta$. Месторождение Додо расположилось ближе всех к Астафьевскому месторождению, но еще на значительном удалении от него. Следовательно, поставленная задача имеет одно решение: по признаку «форма 
кристаллов» оцениваемые проявления отличаются от Астафьевского месторождения и близки к месторождению Желанное

Далее перейдем к построению морфогенетической диаграммы кварца. В качестве её обоснования примем положения, разработанные в предшествующих наших публикациях [4-8]. В тезисной форме они сводятся к следующему.

- Форма кристаллов какого-либо минерального вида при прочих благоприятных условиях однозначно определяется пересыщением. Bсе другие физико-химические факторы среды, как-то температура, давление, химические потенциалы подвижных компонентов влияют на форму опосредовано через изменение пересыщения.

- Морфометрическая функция (F-функция) как интегральная мера формы кристалла зависит от пересыщения, а потому может служить его мерой. На качественном уровне установлено [4, 6], что F-функция снижается с падением пересыщения. Математическое выражение этой зависимости не найдено.

Таблица 2. Расчет переменных параметров и F-функции кристаллов кварца сравниваемых объектов.

\begin{tabular}{|c|c|c|c|c|c|c|c|c|c|}
\hline \multirow{2}{*}{ Объект } & \multirow{2}{*}{$\begin{array}{l}\text { №, } \\
\text { п/п }\end{array}$} & \multicolumn{4}{|c|}{ Измеряемые величины, мм } & \multicolumn{4}{|c|}{ Рассчетные параметры } \\
\hline & & $L m$ & $c$ & $d k$ & $d g$ & $x$ & $y$ & $\eta$ & $F(x, y)$ \\
\hline \multirow{8}{*}{$\begin{array}{l}\text { Астафьевское } \\
\text { месторождене }\end{array}$} & 1 & 302 & 29 & 0 & & 5.2 & 0 & 1 & 7.52 \\
\hline & 2 & 66 & 11 & 0 & & 3.0 & 0 & 1 & 6.59 \\
\hline & 3 & 81 & 15 & 0 & & 2.7 & 0 & 1 & 6.44 \\
\hline & 4 & 60 & 12.5 & 0 & & 2.4 & 0 & 1 & 6.29 \\
\hline & 5 & 72 & 16.5 & 0 & & 2.2 & 0 & 1 & 6.18 \\
\hline & 6 & 64 & 16 & 0 & & 2.0 & 0 & 1 & 6.08 \\
\hline & 7 & 114 & 7.1 & 0 & & 8.0 & 0 & 1 & 8.45 \\
\hline & среднее & & & & & $0.8^{*}$ & - & 1.00 & 5.41 \\
\hline \multirow{4}{*}{ Горбуновское } & 1 & 320 & 32 & 24 & 33 & 5.0 & 0.73 & 0.27 & 7.52 \\
\hline & 2 & 218 & 32 & 25 & 33 & 3.4 & 0.77 & 0.23 & 6.88 \\
\hline & 3 & 223 & 31 & 23 & 32 & 3.6 & 0.73 & 0.27 & 6.96 \\
\hline & среднее & & & & & $0.8^{*}$ & 0.74 & 0.26 & 5.59 \\
\hline \multirow{6}{*}{ Ольховочное } & 1 & 192 & 16 & 11 & 16 & 6 & 0.67 & 0.33 & 7.87 \\
\hline & 2 & 114 & 15 & 10 & 15 & 3.8 & 0.65 & 0.35 & 7.04 \\
\hline & 3 & 282 & 30 & 18 & 31 & 4.7 & 0.59 & 0.41 & 7.39 \\
\hline & 4 & 121 & 9 & 6 & 9 & 6.7 & 0.65 & 0.35 & 8.10 \\
\hline & 5 & 168 & 14 & 9 & 14 & 6 & 0.63 & 0.37 & 7.87 \\
\hline & среднее & & & & & $0.8^{*}$ & 0.64 & 0.36 & 5.57 \\
\hline \multirow{4}{*}{ Вандаманга } & 1 & 228 & 19 & 11 & 17 & 6.0 & 0.65 & 0.35 & 7.87 \\
\hline & 2 & 160 & 20 & 10 & 15 & 4 & 0.66 & 0.34 & 7.12 \\
\hline & 3 & 86 & 12 & 9 & 14.0 & 3.6 & 0.63 & 0.37 & 6.95 \\
\hline & среднее & & & & & $0.8^{*}$ & 0.65 & 0.35 & 5.57 \\
\hline
\end{tabular}

* Примечание: средние значения F-функции определены для приведенного удлинения призматического пояса кристалла $x=0.8$ 
- Автором введено понятие условного относительного пересыщения $\left(\Delta \mathrm{C}^{\circ}\right)$, определяемого по формуле:

$$
\Delta C^{0}=\frac{F}{F_{0}}-1,
$$

где $\mathrm{F}_{0}$ - универсальная кристаллографическая постоянная, равная поверхности шара, объем которого равен $1 . \mathrm{F}_{0}=4.836 \ldots$

При бесконечно малом пересыщении форма кристаллов кубической сингонии стремится к шару. Форма головок кристаллов гетерометричных сингоний стремится к полусфере [6].

Морфогенетическая диаграмма кристаллов кварца рассчитывается по формулам $(13,14)$ и строится в координатах $\eta-\Delta \mathrm{C}^{\circ}$. На построенной таким образом диаграмме (рис. 4) выделены три разной информативности поля.

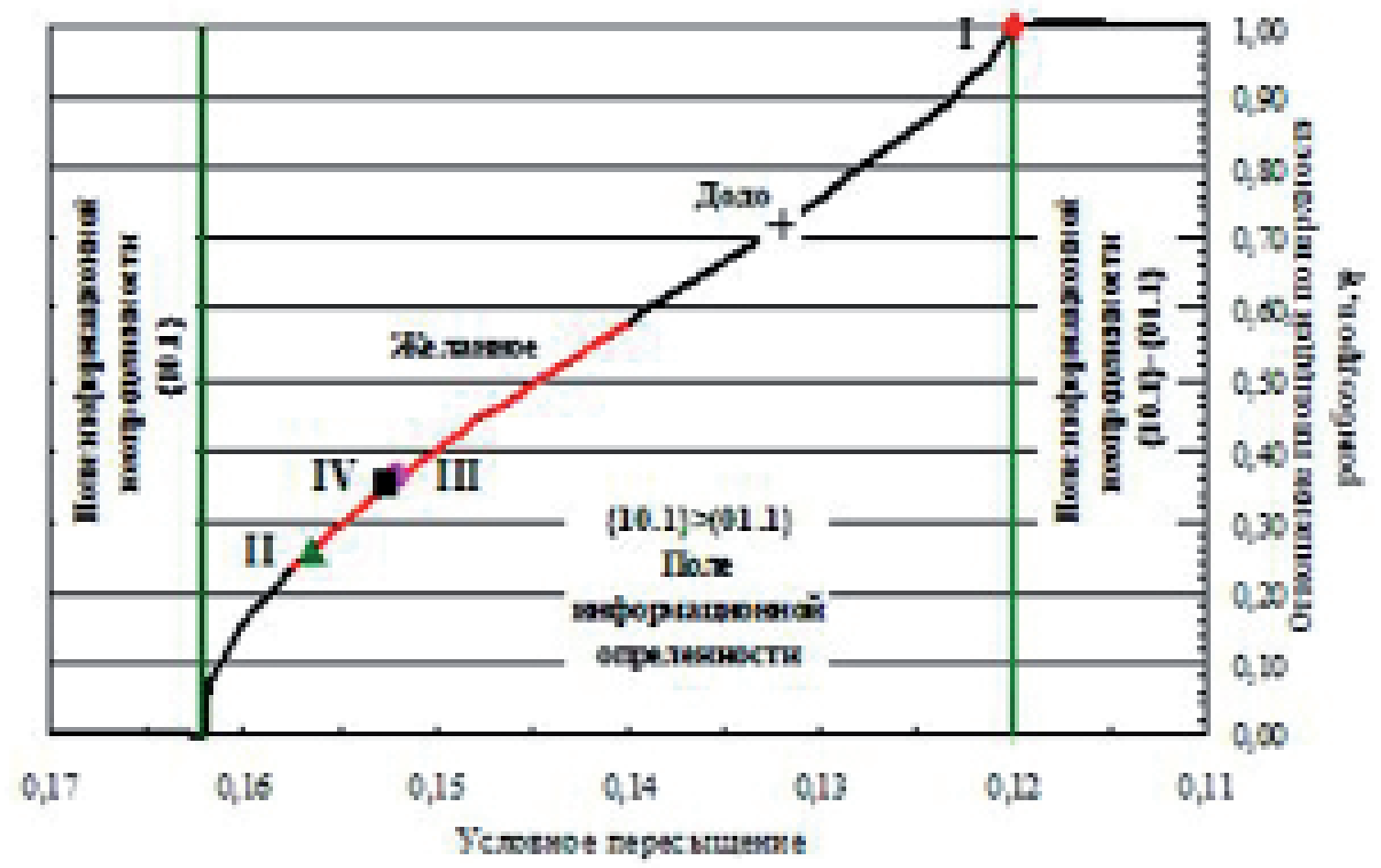

Рис.4. Морфогенетическая диаграмма кристаллов квариа.

Центральное поле, названное полем информационной определенности, заключено в области изменения переменой $0<\eta<1$. и условного пересыщения $0.12<\Delta \mathrm{C}^{\circ}<0.162$. В пределах этого поля по отношению площади граней отрицательного ромбоэдра к площади граней положительного ромбоэдра ( $\eta)$ мы можем определить условное пересыщение.

Левое и правое одномерные поля представлены на графике горизонтальными отрезками кривой и названы полями информационной неопределенности. Правое из них заключено в области: $\eta=1 ; \Delta \mathrm{C}^{\circ} \leq 0.12$, а левое в области: $\eta=0 ; \Delta \mathrm{C}^{\circ} \geq 0.162$. По форме кристаллов, в огранении которых основ- 
ные ромбоэдры равны по площади их поверхностей, можно судить лишь о верхней границе условного пересыщения. В том случае если головки кристаллов образованы гранями только положительного ромбоэдра можно определить лишь нижнюю границу условного пересыщения.

На морфогенетическую диаграмму вынесены те же объекты, что показаны на рисунке 3. Астафьевское месторождение расположилось в правом поле информационной неопределенности, из чего можно заключить, что в сравнении с другими объектами оно образовалось в условиях более низкого пересыщения $\left(\Delta \mathrm{C}^{\circ} \leq 0.12\right)$. При более высоком пересыщении формировалось месторождение Додо $\left(\Delta \mathrm{C}^{\circ}=0.132\right)$. Месторождение Желанное образовалось в условиях снижения пересыщения от 0.158 до 0.14. Для проявлений Горбуновское, Ольховочное и Вандаманга характерны наиболее высокие пересыщения от 0.152 до 0.156.

Введенное понятие «условное пересыщение», если строго следовать предлагаемому алгоритму его определения, можно использовать для сравнительного анализа условий образования любых минералов независимо от их генезиса. Единственным ограничением при этом является требования соответствия формы кристаллов этих минералов классу их симметрии.

\section{Комбинация не коррелятивных ромбоэдров вида $\left\{h_{1} 0 . l_{1}\right\} /\left\{0 k_{2} \cdot l_{2}\right\}$}

Для нахождения уравнения морфометрической функции данной комбинации воспользуемся общей формулой $F$-функции [4], изменив её применительно к условиям задачи.

$$
F\left(x, \rho_{i}\right)=\left[2 \sum_{i=1}^{2} S_{i}\left(x, \rho_{i}\right) n_{i}\left(x, \rho_{i}\right)\right]^{-\frac{2}{3}} 6 \sum_{i=1}^{2} S_{i}\left(x, \rho_{i}\right),
$$

где $S_{i}$ - площадь грани $i$-го ромбоэдра, $x$ - независимая переменная, $n_{i}-$ нормаль к грани $i$-го ромбоэдра, $\rho_{1}$ - полярная координата грани $i$-го ромбоэдра.

Изменение формы и площади граней исследуем с помощью кинематической схемы (рис. 5). В соответствии с этой моделью, изменение формы кристалла происходит за счет нормального роста граней $R 1$ и тангенциального разрастания граней $R 2$.

Выразим все элементы кристалла через независимую переменную $(x)$ и параметр приведения, в качестве которого примем длину $\left(c_{1}\right)$ ребра ромбоэдра 1 в исходном положении. Для этого используем вертикальный разрез, проходящий через диагонали граней $d_{1}$ и $d_{2}$ и ребра $c_{1}$ и $c_{2}$ кристалла (рис. 5a). В качестве независимой переменной примем отношение изменяющейся длины отрезка диагонали $\left(d_{2}\right)$, появляющемся в процессе роста кристалла, к постоянной длине этого отрезка в точке полного перерождения его формы в ромбоэдр 2. Тогда в точке $I x_{i}=k i / b k$ и $k_{i}=x_{i}(b k)$.

Грань $R 2$ зарождается на вершине $k$ первого ромбоэдра и тангенциально разрастается в виде равнобедренного треугольника до того момента $\left(x_{1}\right)$, когда последний двумя нижними вершинами не коснется смежных сторон пол- 


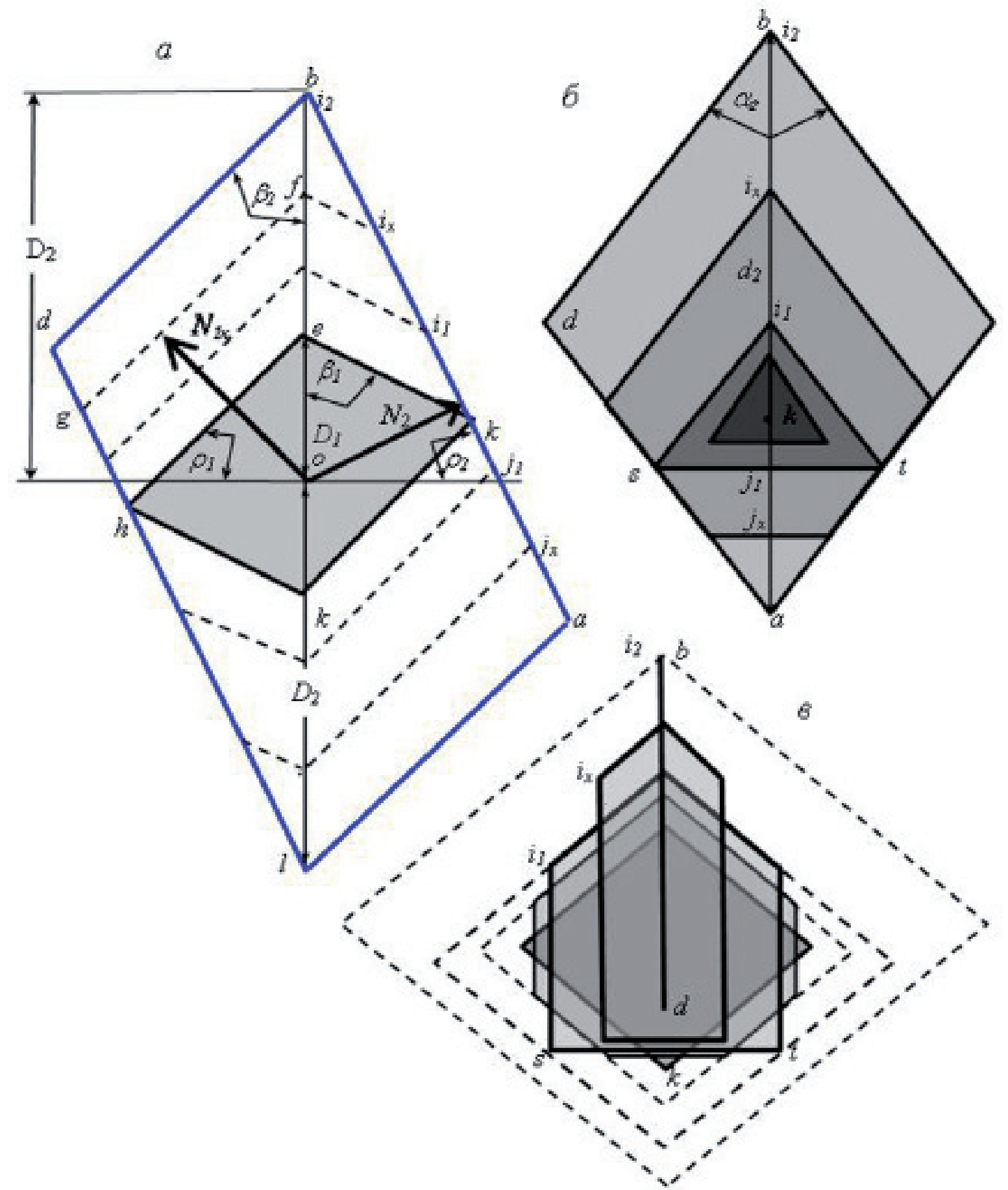

Рис. 5. Кинематическая схема перерождения ромбоэдра $R_{1}$ в ромбоэдр $R_{2}$. $a$ - в разрезе по (11.0), 6 - кинематика грани $R_{2}$, в - кинематика грани $R_{I}$.

ной грани в точках $s$ и $t$. Изменение площади грани до этого момента описывается выражением:

$$
S_{2}^{x_{1}}=(k i+k j)^{2} \operatorname{tg} \frac{\alpha_{2}}{2}=((b k) x+(a k) x)^{2} \operatorname{tg} \frac{\alpha_{2}}{2}=d_{2}^{2} x^{2} \operatorname{tg} \frac{\alpha_{2}}{2}
$$

В момент 1 происходит изменение формы грани $R 2$ с треугольной на ромбическую с усеченной нижней вершиной ромба. Эта форма сохраняется до $x=1$, при котором грань приобретает конечную форму полного ромба. 
Значение аргумента $x_{1}$, при котором меняется форма грани, определяется из соотношения

$$
k i+k j=\frac{a i}{2}, \rightarrow d_{2} x_{1}=\frac{d_{2}-(b k)\left(1-x_{1}\right)}{2} ; \rightarrow x_{1}=\frac{d_{2}-(b k)}{2 d_{2}-(b k)}
$$

От момента $x=x_{1}$ до момента $x=1$ изменение площади грани описывается выражением:

$$
S_{2}^{I I}=\left\{\left[d_{2}-(b k)(1-x)\right] \frac{\operatorname{Sin} \frac{\alpha_{2}}{2}}{\operatorname{Sin} \alpha_{2}}\right\}^{2} \operatorname{Sin} \alpha_{2}-[(a k)(1-x)]^{2} \operatorname{tg} \frac{\alpha_{2}}{2} \text { npu } x_{1} \leq x \leq 1
$$

Грань $R 1$ меняет свою форму в соответствии с чертежом (рис.5.в) и в конце процесса вырождается в ребро ромбоэдра $R 2$. Изменение формы и площади грани описывается уравнением:

$$
S_{1}=\mid \begin{aligned}
& \left.\frac{(f g)^{2}}{2}-(f g-f g)^{2}\right] \operatorname{tg} \frac{\alpha_{1}}{2}-\left(\frac{d_{2} x}{\operatorname{Cos} \frac{\alpha_{2}}{2}}\right)^{2} \frac{\operatorname{tg} \frac{\alpha_{1}}{2}}{2} \quad \text { nрu } 0 \leq x \leq x_{1} \\
& (s t)(s i)+\frac{(s t)^{2}}{4 \operatorname{tg} \frac{\alpha_{1}}{2}} \quad \text { nри } x_{1} \leq x \leq 1
\end{aligned}
$$

Выразим все элементы, входящие в формулы (16-19), через параметр приведения $\boldsymbol{c}$

$$
\begin{aligned}
& (b k)=c_{1} \frac{\operatorname{Sin} \beta_{1}}{\operatorname{Cos} \rho_{2}} ; 2 D_{1}=c_{1} \frac{\operatorname{Cos}\left(\beta_{1}-\rho_{1}\right)}{\operatorname{Cos} \rho_{1}} ; \quad D_{2}=(b e)+D_{1}=c_{1}\left[\frac{\operatorname{Sin}\left(\rho_{2}+\beta_{1}-90^{\circ}\right)}{\operatorname{Cos} \rho_{2}}+\frac{\operatorname{Cos}\left(\beta_{1}-\rho_{1}\right)}{2 \operatorname{Cos} \rho_{1}}\right] \\
& d_{2}=2 D_{2} \frac{\operatorname{Sin} \beta_{2}}{\operatorname{Cos}\left(\beta_{2}-\rho_{2}\right)}=c_{1}\left[\frac{\operatorname{Sin}\left(\rho_{2}+\beta_{1}-90^{\circ}\right)}{\operatorname{Cos} \rho_{2}}+\frac{\operatorname{Cos}\left(\beta_{1}-\rho_{1}\right)}{2 \operatorname{Cos} \rho_{1}}\right] \frac{2 \operatorname{Sin} \beta_{2}}{\operatorname{Cos}\left(\beta_{2}-\rho_{2}\right)} ;
\end{aligned}
$$

Угол $(\beta)$ между объемной диагональю и ребром и угол $(\alpha)$ между ребрами ромбоэдра определяются из геометрических отношений элементов ромбоэдра по формулам:

$$
\alpha_{i}=2 \operatorname{arctg}\left(\sqrt{3} \operatorname{Cos} \rho_{i}\right), \quad \beta_{i}=\operatorname{arctg}\left(\frac{2}{\operatorname{tg} \rho_{i}}\right)
$$

Для сокращения записи конечных формул введем следующие обозначения тригонометрических функций (20):

$$
\begin{aligned}
& \frac{\operatorname{Sin} \beta_{1}}{\operatorname{Cos} \rho_{2}}=A ; \frac{\operatorname{Sin}\left(\rho_{2}+\beta_{1}-90^{0}\right)}{\operatorname{Cos} \rho_{2}}+\frac{\operatorname{Cos}\left(\beta_{1}-\rho_{1}\right)}{2 \operatorname{Cos} \rho_{1}}=B ; \frac{\operatorname{Sin} \beta_{2}}{\operatorname{Cos}\left(\beta_{2}-\rho_{2}\right)}=C ; \frac{\operatorname{Sin} \frac{\alpha_{2}}{2}}{\operatorname{Sin} \alpha_{2}}=D ; \\
& \frac{\operatorname{Sin}\left(\rho_{2}+\beta_{1}-90^{0}\right)}{\operatorname{Cos} \rho_{2}}=E ; \quad \frac{\operatorname{Cos} \rho_{2}}{\operatorname{Cos}\left(\beta_{2}-\rho_{2}\right)}=F ; \quad \frac{\operatorname{Sin} \beta_{1}}{\operatorname{Cos}\left(\beta_{1}-\rho_{1}\right)}=G
\end{aligned}
$$


Выразим переменные линейные элементы кристалла, входящие в $(15,19)$ через параметр приведения $\boldsymbol{c}$, независимую переменную $x$ и тригонометрические функции (22).

$$
\begin{gathered}
(s t)=2(a k)(1-x) \operatorname{tg} \frac{\alpha_{2}}{2}=2 c_{1}[2 B C-A](1-x) \operatorname{tg} \frac{\alpha_{2}}{2} \\
(s i)=\left[d_{2}-(b k)(1-x)\right] \frac{\operatorname{Sin} \frac{\alpha_{2}}{2}}{\operatorname{Sin} \alpha_{2}}=c[2 B C-A(1-x)] D \\
(b f)=(b i) \frac{\operatorname{Sin}\left(\rho_{2}+\beta_{1}-90^{\circ}\right)}{\operatorname{Sin} \beta_{1}}=(b k)(1-x) \frac{\operatorname{Sin}\left(\rho_{2}+\beta_{1}-90^{\circ}\right)}{\operatorname{Sin} \beta_{1}}=c(1-x) A E \\
n_{1}=\left(D_{2}-(b f)\right) \operatorname{Cos} \rho_{1}=c_{1}[B-(1-x) A E] \operatorname{Cos} \rho \quad(26) ; \quad n_{2}=D_{2} \operatorname{Cos} \rho_{2} ; \\
(f g)=\left[2 D_{2}-(b f)\right] \frac{\operatorname{Cos} \rho_{2}}{\operatorname{Cos}\left(\beta_{2}-\rho_{2}\right)}=c_{1}[2 B-(1-x) A E] F ; \\
(f g)=2\left[D_{2}-(b f)\right] \frac{\operatorname{Sin} \beta_{1}}{\operatorname{Cos}\left(\beta_{1}-\rho_{1}\right)}=2 c_{1}[B-(1-x) A E] G
\end{gathered}
$$

Подставив полученные выражения в (16-19), получим формулу для вычисления площади граней ромбоэдров.

$$
\begin{gathered}
S_{1}=c_{1}^{2} \mid \begin{array}{c}
S_{1}^{I}=\left\langle 2 G^{2}[B-A E(1-x)]^{2}-\{2 G[B-A E(1-x)]-F[2 B-A E(1-x)]\}^{2}\right\rangle \operatorname{tg} \frac{\alpha_{1}}{2}-\left[\frac{2 B C x}{\operatorname{Cos} \frac{\alpha_{2}}{2}}\right]^{2} \frac{\operatorname{tg} \frac{\alpha_{1}}{2}}{2} \\
n p u 0 \leq x \leq \frac{2 B C-A}{4 B C-A} \\
S_{1}^{I I}=2[2 B C-A](1-x)[2 B C-A(1-x)] D+\frac{\left[(2 B C-A)(1-x) \operatorname{tg} \frac{\alpha_{2}}{2}\right]^{2}}{\operatorname{tg} \frac{\alpha_{1}}{2}} n p u \quad \frac{2 B C-A}{4 B C-A} \leq x \leq 1
\end{array} \\
S_{2}=c_{1}^{2} \mid \begin{array}{ll}
S_{2}^{I}=4 B^{2} C^{2} x^{2} \operatorname{tg} \frac{\alpha_{2}}{2} \quad n p u \quad 0 \leq x \leq \frac{2 B C-A}{4 B C-A} \\
S_{2}^{I I}=\{[2 B C-A(1-x)] D\}^{2} \operatorname{Sin} \alpha_{2}-[2 B C-A(1-x)]^{2} \operatorname{tg} \frac{\alpha_{2}}{2} \quad \text { npu } \frac{2 B C-A}{4 B C-A} \leq x \leq 1
\end{array}
\end{gathered}
$$

Подставив $(30,31)$ в $(15)$ получим искомую формулу морфометрической функции комбинации двух не коррелятивных ромбоэдров. 


$$
F=\mid \begin{array}{ll}
2\left(S_{1}^{I} n_{1}+S_{2}^{I} n_{2}\right)^{-\frac{2}{3}} 6\left(S_{1}^{I}+S_{2}^{I}\right) & \text { при } 0 \leq x \leq \frac{2 B C-A}{4 B C-A} \\
2\left(S_{1}^{I I} n_{1}+S_{2}^{I I} n_{2}\right)^{-\frac{2}{3}} 6\left(S_{1}^{I I}+S_{2}^{I I}\right) & \text { nри } \frac{2 B C-A}{4 B C-A} \leq x \leq 1
\end{array}
$$

Использование выведенной формулы $F$-функции комбинации двух не коррелятивных ромбоэдров продемонстрируем на примере кристаллов кальцита.

\section{Пример.}

Задача: рассчитать формулу и построить графики морфометрических функций комбинационной пары не коррелятивных ромбоэдров, встречающихся в огранении кристаллов исландского шпата Сибирской платформы [3].

Решение задачи. Сначала из ряда возможных ромбоэдров данного класса выделим совместимые пары, используя критерий совместимости $\left(\mathrm{K}_{a}\right)$, рассчитываемый из условий, налагаемых кинематической моделью; $\rho_{1}<\rho_{2}$ и $\left(90^{\circ}-\rho_{1}\right)=\beta_{2}$. Из второго условия и (21) для совместимых ромбоэдров получим

$$
\beta_{2}=\operatorname{arctg}\left(\frac{2}{\operatorname{tg} \rho_{2}}\right) \rightarrow \operatorname{tg}\left(\frac{\pi}{2}-\rho_{1}\right) \operatorname{tg} \rho_{2}=2
$$

Для несовместимых ромбоэдров $\mathrm{K}_{a} \neq 2$ из таблицы 3 видно, что из возможных 8 пар не коррелятивных гетерозональных ромбоэдров совместимыми являются три: $\{10.1\} /\{02.1\} ;\{01.2\} /\{10.1\} ;\{02.1\} /\{40.1\}$. Для этих пар $\mathrm{K}_{a}=2$.

Таблица 3. Критерий совместимости не коррелятивных гетерозональных ромбоэдров в огранении кристаллов кальцита.

\begin{tabular}{|c|c|c|c|c|c|}
\hline & R, отр. & $\{01.2\}$ & $\{03.2\}$ & $\{02.1\}$ & $\{05.4\}$ \\
\hline R, пол. & r, рад. & 0.4582 & 0.9765 & 1.1015 & 0.88925 \\
\hline$\{40.1\}$ & 1.32296 & 8.01 & 2.67 & $\mathbf{2 . 0 0}$ & 3.21 \\
\hline$\{10.1\}$ & 0.77842 & $\mathbf{2 . 0 0}$ & 1.50 & $\mathbf{2 . 0 0}$ & 1.25 \\
\hline
\end{tabular}

Для пар совместимых ромбоэдров вычислим постоянные, входящие в выражения (30, 31), и представим их в табличном виде (табл. 4).

Таблица 4.

\begin{tabular}{|c|c|c|c|c|c|c|c|c|c|}
\hline $\mathrm{R} 1 \backslash \mathrm{R} 2$ & $\mathrm{~A}$ & $\mathrm{~B}$ & $\mathrm{C}$ & $\mathrm{D}$ & $\mathrm{E}$ & $\mathrm{F}$ & $\mathrm{G}$ & $\alpha_{1}$, рад. & $\alpha_{2}$, рад. \\
\hline$\{10.1\} \backslash\{02.1\}$ & 1.98 & 1.98 & 0.75 & 0.64 & 0.67 & 0.48 & 0.95 & 1.8 & 1.54 \\
\hline$\{01.2\} \backslash\{10.1\}$ & 1.36 & 1.08 & 0.95 & 0.79 & 0.53 & 0.75 & 1.51 & 2.0 & 1.8 \\
\hline$\{02.1\} \backslash\{40.1\}$ & 2.90 & 3.17 & 0.69 & 0.54 & 0.73 & 0.37 & 0.75 & 1.54 & 0.8 \\
\hline
\end{tabular}

Подставив значения постоянных из таблицы 4 в выражения площади граней и нормалей к ним, а последние в (32) и произведя необходимые ал- 
гебраические действия, получим формулы F-функции для рассматриваемых парных комбинаций ромбоэдров.

$$
\begin{gathered}
F=\mid \begin{array}{l}
\{10.1\} \backslash\{02.1\} \\
\left(0.926+5.548 x+11.124 x^{2}-17,54 x^{3}\right)^{-\frac{2}{3}} 6\left(0.98+3.918 x-2,347 x^{2}\right) \quad n p u \quad 0 \leq x \leq 0.25 \\
\left(0,69+8,314 x-2,77 x^{3}\right)^{-\frac{2}{3}} 6\left(1,081+3,08 x-0,698 x^{2}\right) \quad n p u \quad 0.25 \leq x \leq 1
\end{array} \\
F=\mid \begin{array}{l}
\left(0.58+3,506 x+7,016 x^{2}-11,13 x^{3}\right)^{-\frac{2}{3}} 6\left(0.904+3.645 x-3,476 x^{2}\right) \quad n p u \quad 0 \leq x \leq 0.25 \\
\left(0,436+5,259 x+0,014 x^{2}-1,754 x^{3}\right)^{-\frac{2}{3}} 6\left(1,075+2,285 x-0,782 x^{2}\right) \quad n p u \quad 0.25 \leq x \leq 1
\end{array} \\
F=\mid \begin{array}{l}
\{02.1\} \backslash\{40.1\} \\
\left(0.919+5,56 x+11,34 x^{2}-17,679 x^{3}\right)^{-\frac{2}{3}} 6\left(0.967+3.908 x-1,093 x^{2}\right) \quad n p u \quad 0 \leq x \leq 0.25 \\
\left(0,69+8,345 x-2,789 x^{3}\right)^{-\frac{2}{3}} 6\left(1,011+3,58 x-0,568 x^{2}\right) \quad n p u \quad 0.25 \leq x \leq 1
\end{array}
\end{gathered}
$$

Морфометрическая диаграмма для комбинационных пар ромбоэдров кальцита, построенная по уравнениям (34-36), представлена на рисунке 6. Основными габитусными формами ромбоэдрических кристаллов кальцита являются $\{10.1\}$ и \{02.1\}. На график F-функции этой пары вынесены две формы кальцита, встречающиеся на месторождениях исландского шпата Сибирской платформы [3]. Одна из них образована комбинацией двух ромбоэдров при доминировании ромбоэдра $\{10.1\}$, морфометрическая функция её равна $F(x=0.3)=5.47$. Вторая форма характеризуется абсолютным доминированием ромбоэдра $\{02.1\}$, морфометрическая функция её равна $F(x=0.9)=5.87$.

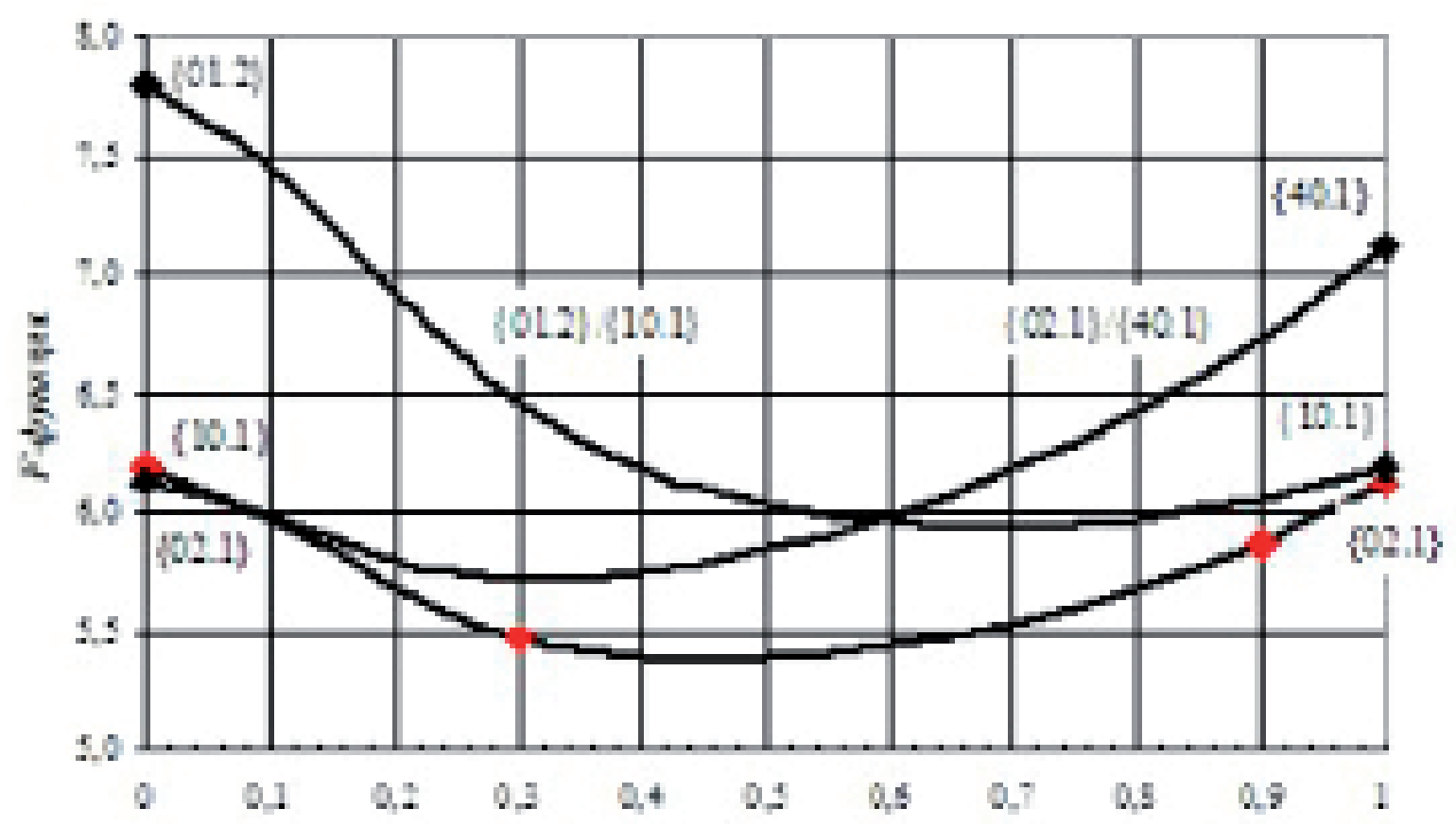

Рис. 6. Морфометрическая диаграмма ромбоэдрических кристаллов кальцита. 
Морфогенетическую диаграмму кальцита построим в координатах $\Delta \mathrm{C}^{\circ}-F$ для пары габитусных ромбоэдров $\{10.1\} /\{02.1\}$. По формуле (14) определим пересыщение для одиночных ромбоэдров $\{10.1\}:\left(\Delta \mathrm{C}^{\circ}{ }_{\{10.1\}}=6.187 / 4.836-1=0.28\right)$; $\left.\Delta \mathrm{C}^{\circ}{ }_{\{02.1\}}=6.136 / 4.836-1=0.268\right)$. Допускаем, что с течением времени пересыщение изменяется линейно, что позволяет проградуировать ось абсцисс и построить морфогенетическую диаграмму (рис. 7).

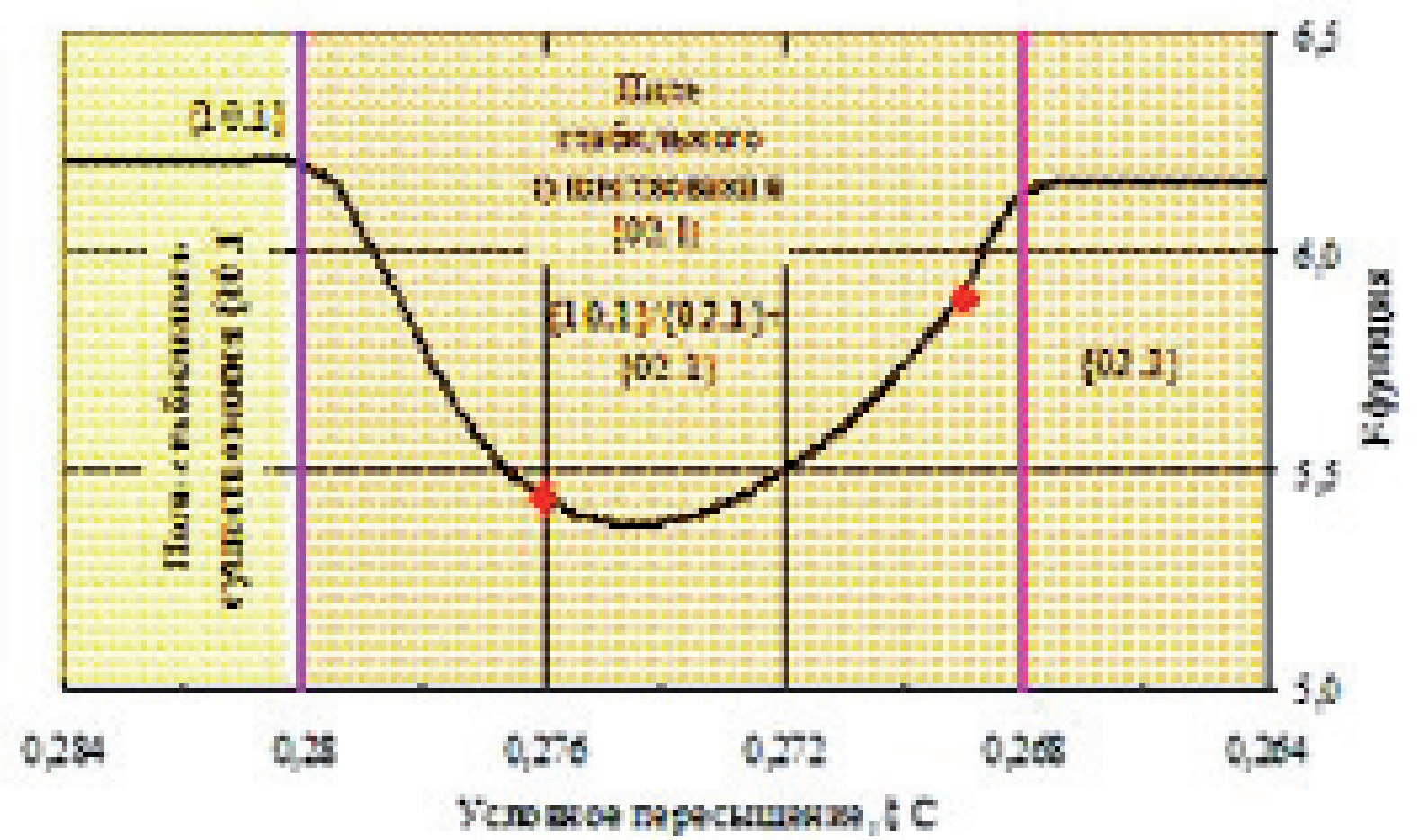

Рис. 7. Морфогенетическая диаграмма кристаллов кальциита ромбоэдрического габитуса.

Выделим на диаграмме поля равновесного роста рассматриваемых ромбоэдров и определим их габитусные формы в пределах каждого поля. В соответствии с феноменологической теорией морфогенеза кристаллов [8] каждая закрытая простая форма является равновесной в определенном интервале пересыщений. Условное пересыщение 0.28 принимаем за правую границу поля равновесного роста ромбоэдра $\{10.1\}$. Вправо от $\Delta \mathrm{C}^{\circ}=0.28$, в направлении уменьшения пересыщения, располагается поле равновесного роста ромбоэдра $\{02.1\}$. В этом поле могут сосуществовать две генерации габитусных форм кальцита. Ранняя генерация представлена комбинацией ромбоэдров $\{10.1\} /\{02.1\}$. При этом ромбоэдр $\{10.1\}$ метастабильный, с течением времени вырождающийся. Поздняя генерация представлена ромбоэдром $\{02.1\}$. С учетом принятого допущения о линейном изменении пересыщения метастабильный в этом поле ромбоэдр $\{10.1\}$ должен окончательно выродиться на границе $\Delta \mathrm{C}^{\circ}=0.268$. Правее этой границы в огранении кристаллов должен присутствовать только ромбоэдр $\{02.1\}$. Анатомическая картина реальных ромбоэдрических кристаллов исландского шпата Сибирской плат- 
формы показывает, что ядерные части кристаллов образованы пирамидами роста граней ромбоэдра $\{10.1\}[3]$. Это указывает на то, что кристаллизация шпата происходила в условиях постепенного снижения пересыщения гидротермального раствора. В соответствии с этим фактом на морфогенетической диаграмме использован обратный порядок изменения пересыщения. Принятый порядок соответствует также направлению течения времени кристаллизации.

Провести морфометрический анализ других комбинационных форм тригональной сингонии с участием скаленоэдров и трапецоэдров весьма сложно. Автор предлагает лабораторный метод определения $F$-функции таких комбинаций, простая методика которого описана в [7]. Для этого необходимо располагать коллекцией из минимум трех (желательно больше) кристаллов с разным соотношением габитусных форм. Морфометрическая диаграмма при этом может быть построена путем аппроксимации эмпирических точек показательной или степенной функцией.

\section{Список литературы}

1. Балицкий В.С. Экспериментальное изучение геохимических условий формирования кристаллов кварца. М., 1971.

2. Дэна Дж., Дэна Э. С., Фрондель К. Система минералогии. Минералы кремнезема. М.: МИР, 1966.

3. Скропышев А.В., Кукуй А.Л. Исландский шпат. Л.: Недра, 1973.

4. Страшненко Г.И. Теоретическое обоснование интегрального количественного показателя формы кристаллов. Тр. XI Всерос. (с межд. участием) науч. школы «Математические исследования в естественных науках» // Апатиты, 11-12 нояб. 2016 г. Апатиты: Изд-во К \& М, 2014. С. 48-119.

5. Страшненко Г.И. Морфометрический анализ простых и квазипростых форм кристаллов тригональной и гексагональной сингоний // Тр. XIII Всеpoc. (с межд. участием) Ферсмановской научн. сессии, посв. 50-летию Дня геолога. Апатиты, 4-5 апр. 2016 г. Апатиты: Изд-во К \& М, 2016. С. 310-323. 6. Страшненко Г.И. Равновесная форма кристаллов средних и низших категорий симметрии при бесконечно малых пересыщениях // Tp. XIII Bceроссийской (с международным участием) научной школы «Математические исследования в естественных науках» Апатиты, 17-18 окт. 2016 г. Апатиты: Изд-во К \& М, 2016. С. 85-96.

7. Страшненко Г. И. Лабораторный метод определения морфометрической функции сложно ограненных кристаллов // Тр. XIII Всерос. (с межд. участием) научной школы «Математические исследования в естественных науках» Апатиты, 17-18 окт. 2016 г. Апатиты: Изд-во К \& М, 2016. С. 97-98.

8. Страшненко Г.И. Морфометрия и морфогенез кристаллов. LAP LAMBERT Acad. Publ., 2016.

9. Шаскольская М. П. Кристаллография. М.: «Высшая школа», 1976. 